
Preprints des Instituts für Mathematik der Universität Potsdam 

Preprints des Instituts für Mathematik der Universität Potsdam 5 (2016) 4

\section{Variational Primitive of a Differential Form}


Bibliografische Information der Deutschen Nationalbibliothek

Die Deutsche Nationalbibliothek verzeichnet diese Publikation in der Deutschen Nationalbibliografie; detaillierte bibliografische Daten sind im Internet über http://dnb.dnb.de abrufbar.

\section{Universitätsverlag Potsdam 2016}

http://verlag.ub.uni-potsdam.de/

Am Neuen Palais 10, 14469 Potsdam

Tel.: +49 (0)331 9772533 / Fax: 2292

E-Mail: verlag@uni-potsdam.de

Die Schriftenreihe Preprints des Instituts für Mathematik der Universität Potsdam wird herausgegeben vom Institut für Mathematik der Universität Potsdam.

ISSN (online) 2193-6943

Kontakt:

Institut für Mathematik

Karl-Liebknecht-Straße 24/25

14476 Potsdam

Tel.: +49 (0)331977 1499

WWW: http://www.math.uni-potsdam.de

Titelabbildungen:

1. Karla Fritze | Institutsgebäude auf dem Campus Neues Palais

2. Nicolas Curien, Wendelin Werner | Random hyperbolic triangulation

Published at: http://arxiv.org/abs/1105.5089

Das Manuskript ist urheberrechtlich geschützt.

Online veröffentlicht auf dem Publikationsserver der Universität Potsdam

URL https://publishup.uni-potsdam.de/opus4-ubp/frontdoor/index/index/docId/8922

URN urn:nbn:de:kobv:517-opus4-89223

http://nbn-resolving.de/urn:nbn:de:kobv:517-opus4-89223 


\title{
VARIATIONAL PRIMITIVE OF A DIFFERENTIAL FORM
}

\author{
AMMAR ALSAEDY
}

This paper is dedicated to my teacher N. Tarkhanov on the occasion of his 60 th birthday.

ABstract. In this paper we specify the Dirichlet to Neumann operator related to the Cauchy problem for the gradient operator with data on a part of the boundary. To this end, we consider a nonlinear relaxation of this problem which is a mixed boundary problem of Zaremba type for the p-Laplace equation.

\section{Contents}

Introduction

1. The Cauchy problem

2. The Euler equations

3. Existence of variational solution

4. The Dirichlet to Neumann operator

References

4

6

8

\section{INTRODUCTION}

The classical Cauchy problem for the gradient operator has attracted considerable attention both in geometry and analysis of the last century. It reads as follows. Let $\mathcal{X}$ be a bounded domain with smooth boundary in $\mathbb{R}^{n}$ and $\mathcal{S}$ a non empty open piece of the boundary surface $\partial \mathcal{X}$. (The case $\mathcal{S}=\partial \mathcal{X}$ is included as well.) Consider the Cauchy problem

$$
\left\{\begin{aligned}
\nabla u & =f \quad \text { in } \mathcal{X} \\
u & =u_{0} \quad \text { at } \mathcal{S}
\end{aligned}\right.
$$

in $\mathcal{X}$ with data $f \in L^{p}\left(\mathcal{X}, \mathbb{R}^{n}\right)$ and $u_{0} \in W^{1 / p^{\prime}, p}(\mathcal{S})$, where $1<p<\infty$ and $p^{\prime}=p /(p-1)$,

This problem fails to be solvable in general, unless $f$ satisfies some compatibility conditions and $u_{0}$ belongs to a "thin" set of Cauchy data at $\mathcal{S}$, [Tar95]. Since the problem $(0.1)$ is overdetermined, we relax it to the problem of minimising the $L^{p}$-norm

$$
I(u):=\int_{\mathcal{X}}|\nabla u-f|^{p} d x
$$

Date: March 14, 2016.

2010 Mathematics Subject Classification. Primary 35J92; Secondary 35J66, 47A52.

Key words and phrases. Dirichlet to Neumann operator, Cauchy problem, $p$-Laplce operator, calculus of variations. 
over the set $\mathcal{A}$ of all functions $u \in W^{1, p}(\mathcal{X})$ satisfying $u=u_{0}$ at $\mathcal{S}$. A direct computation shows that the Euler-Lagrange equations for the variational problem $I(u) \mapsto \min$ are

$$
\left\{\begin{aligned}
\operatorname{div}\left(|\nabla u-f|^{p-2}(\nabla u-f)\right) & =0 \quad \text { in } \mathcal{X}, \\
u & =u_{0} \text { at } \mathcal{S}, \\
|\nabla u-f|^{p-2}(\nabla u-f, \nu) & =0 \quad \text { at } \partial \mathcal{X} \backslash \mathcal{S},
\end{aligned}\right.
$$

which actually constitute a mixed boundary value problem of Zaremba type [Zar10]. For $f=0$, this mixed problem was investigated in [She13]. The p-Laplace operator is a quasilinear operator that generalises the linear Laplace operator which corresponds to $p=2$. The p-Laplace operator plays an important role in nonlinear potential theory and appears often in physics, [AH96, p. 166].

Our purpose in this paper is to show that the variational problem $I(u) \mapsto \min$ has a unique solution for all data $f \in L^{p}\left(\mathcal{X}, \mathbb{R}^{n}\right)$ and $u_{0} \in W^{1-1 / p, p}(\mathcal{S})$.

When having granted the unique solvability of the problem $(0.2)$, we are in a position to introduce a Dirichlet to Neumann operator for problem (0.1).

The Dirichlet to Neumann operator $\Psi$ is intended to describe the set of all data $u_{0}$ for which the Cauchy problem is solvable, cf. [LT11]. More precisely, $\Psi$ is an operator acting in function spaces on $\mathcal{S}$, such that the range of the Cauchy problem coincides with the zero set of $\Psi$. The relevance of the Dirichlet to Neumann operator to the inverse problem of impedance tomography was understood at least as far as [Cal80]. Nowadays a considerable number of papers are devoted to this operator which study, in particular, its symbol, spectrum, etc.

This paper can be thought of as development to a part of the results of [She13] and [AT14].

\section{The Cauchy problem}

We now return to Cauchy problem (0.1). To this end we identify $\nabla u$ with the exterior derivative of the function $u$, which is denoted by $d u$. If $u \in W^{1, p}(\mathcal{X})$ satisfies the equation $d u=f$ in $\mathcal{X}$, then $d f=0$ weakly in $\mathcal{X}$, for $d^{2}=0$. In other words, the differential equation in (0.1) is solvable only for those differential forms $f$ which are closed. Since the set of closed differential forms in $\mathcal{X}$ is "thin" in an appropriate sense, we pass to a variational formulation of the Cauchy problem. To wit, we look for a solution of the extremal problem $I(u) \mapsto$ min for the functional

$$
I(u):=\int_{\mathcal{X}}|\nabla u-f|^{p} d x
$$

over the set $\mathcal{A}$ of all functions $u \in W^{1, p}(\mathcal{X})$ satisfying $u=u_{0}$ at $\mathcal{S}$. Obviously, every solution of (0.1) minimises the variational problem. The converse assertion is not true.

We mention here that this problem was proved to be solvable for specific choice of the function $f$, see [AT14]. More precisely, for the solvability of the inhomogeneous system $d u=f$ in $\mathcal{X}$ it is necessary that

$$
(f, g)_{L^{2}\left(\mathcal{X}, \mathbb{C}^{n}\right)}=0
$$

for all functions $g \in W^{1, p^{\prime}}\left(\mathcal{X}, \mathbb{R}^{n}\right)$ satisfying $\operatorname{div} g=0$ in $\mathcal{X}$ and $(\nu, g)=0$ at $\partial \mathcal{X}$, where $\nu(x)$ is the unit outward normal vector to the boundary at $x \in \partial \mathcal{X}$. Choosing $g=d^{*} v$, where $v$ is a smooth differential form of degree 2 with compact support in the interior of $\mathcal{X}$ and $d^{*}$ the formal adjoint of the exterior derivative, we conclude 
that $d f=0$ in $\mathcal{X}$. If the de Rham cohomology of $\mathcal{X}$ at step 1 is zero (e.g., if the domain $\mathcal{X}$ is contractible), then the condition $d f=0$ is also sufficient for the existence of a function $U_{0} \in W^{1, p}(\mathcal{X})$ satisfying $d U_{0}=f$ in $\mathcal{X}$. This is a consequence of the ellipticity of the Neumann problem for the de Rham complex, see Section 4.1.3 of [Tar95] and elsewhere. The change of dependent variables $U=u-U_{0}$ reduces then the variational problem $I(u) \mapsto \min$ over $\mathcal{A}$ to the problem treated in [She13]. This proves the unique solvability of the variational problem under the above additional condition. In particular, the mixed problem (0.2) has a unique solution.

In Section 7 of [AT14] we introduced the Dirichlet to Neumann operator to describe those $f$ and $u_{0}$ for which the Cauchy problem (0.1) is solvable.

In this paper we develop the techniques of both [She13] and [AT14] to show that the variational problem $I(u) \mapsto$ min has a unique solution for all data $f \in L^{p}\left(\mathcal{X}, \mathbb{R}^{n}\right)$ and $u_{0} \in W^{1 / p^{\prime}, p}(\mathcal{S})$, not only for those satisfying $d f=0$ in $\mathcal{X}$.

\section{The Euler equations}

Write $m$ for the infimum of $I(u)$ over $u \in \mathcal{A}$. In order that $u \in \mathcal{A}$ may satisfy $I(u)=m$, it is necessary that $u$ would fulfill the so-called Euler-Lagrange equations. These latter could be described as follows.

Pick an arbitrary function $\delta \in C^{\infty}(\mathcal{X})$ vanishing on $\mathcal{S}$. For all $t \in \mathbb{R}$, the variation $u+t \delta$ does not go beyond $\mathcal{A}$ for any $u \in \mathcal{A}$. Therefore, if $I(u)=m$, then the function $F(t)=I(u+t \delta)$ takes on a local minimum at $t=0$. It follows that $t=0$ is a critical point of $F$.

Computing $F^{\prime}(0)$, using the Gauss formula, and applying the main lemma of calculus of variations yields

$$
\left\{\begin{aligned}
\operatorname{div}\left(|\nabla u-f|^{p-2}(\nabla u-f)\right) & =0 \quad \text { in } \mathcal{X} \\
u & =u_{0} \text { at } \mathcal{S} \\
|\nabla u-f|^{p-2}(\nabla u-f, \nu) & =0 \quad \text { at } \partial \mathcal{X} \backslash \mathcal{S}
\end{aligned}\right.
$$

where $\nu$ refers to the outward unit normal vector of the boundary surface. Equations (2.1) constitute a mixed boundary value problem of Zaremba type [Zar10]. The nonlinear differential equation of (2.1) reveals the well-known p-Laplace equation, however, it can be reduced to this latter only in the case where $f$ is closed.

For $p=2$ we just recover the usual Laplacian. For $p>2$ the p-Laplace equation is degenerate elliptic and for $1<p<2$ singular at the points where $\nabla u-f=0$.

As mentioned in Section 1, this mixed problem was treated for specific choice of the 1 -form $f$. More precisely, if $d f=0$ in $\mathcal{X}$ and the first de Rham cohomology of $\mathcal{X}$ vanishes, then problem (2.1) possesses a unique solution $u \in W^{1, p}(\mathcal{X})$, see [AT14].

By a weak solution $u \in W^{1, p}(\mathcal{X})$ of $(2.1)$ is meant any solution of the variational problem $I(u) \rightarrow$ min over $u \in \mathcal{A}$. Even if $u$ takes on its minimum $m$ for some $u \in \mathcal{A}$, the function $u$ need not satisfy (0.1) unless $m=0$. Hence, if (2.1) possesses a weak solution, then for the solvability of the Cauchy problem it is necessary and sufficient that $m=0$. From the construction above, one sees immediately that the Zaremba problem arises naturally in the calculus of variations. 


\section{Existence of VARiational SOLUtion}

In this section we show the unique solvability of the variational problem. To this end we prove some crucial properties of the functional $I(u)$.

We start with the following lemma which elucidates the behaviour of the functional $I(u)$ for large functions $u$.

Lemma 3.1. The functional $I(u)$ is weakly coercive on the space $\mathcal{A}$, i.e., $I(u) \rightarrow \infty$ as $\|u\|_{W^{1, p}(\mathcal{X})} \rightarrow \infty$.

Proof. We first estimate the functional $I(u)$. To wit,

$$
\begin{aligned}
\left(\int_{\mathcal{X}}|\nabla u-f|^{p} d x\right)^{1 / p} & =\|\nabla u-f\|_{L^{p}(\mathcal{X})} \\
& \geq\|\nabla u\|_{L^{p}(\mathcal{X})}-\|f\|_{L^{p}(\mathcal{X})} \\
& =\|\nabla u\|_{L^{p}(\mathcal{X})}-Q
\end{aligned}
$$

where $Q$ is a constant independent of $u$. According to Lemma 1.9.2 of [Mor66], there is a constant $C$ depending only on $\mathcal{X}, p$ and $n$, such that

$$
\|u\|_{W^{1, p}(\mathcal{X})} \leq C\left(\|\nabla u\|_{L^{p}\left(\mathcal{X}, \mathbb{R}^{n}\right)}+\|u\|_{L^{1}(\mathcal{X})}\right)
$$

for all $u \in \mathcal{A}$. (Such estimates are usually referred to as Korn estimates.) It follows that $\|\nabla u\|_{L^{p}(\mathcal{X})} \rightarrow \infty$ as $\|u\|_{W^{1, p}(\mathcal{X})} \rightarrow \infty$.

This implies $I(u) \rightarrow \infty$ as $\|u\|_{W^{1, p}} \rightarrow \infty$, which precisely means that $I(u)$ is coercive.

In order to prove the lower semicontinuity of $I(u)$ and uniqueness later, we need first to show that $I(u)$ is strictly convex.

Lemma 3.2. The functional $I(u)$ is strictly convex on $\mathcal{A}$.

Proof. It is obvious that $\mathcal{A}$ is convex. If we take two different functions $u, v \in \mathcal{A}$, then $t u+(1-t) v=u_{0}$ at $\mathcal{S}$. Hence $t u+(1-t) v \in \mathcal{A}$. We have to show that

$$
I(t u+(1-t) v)<t I(u)+(1-t) I(v)
$$

for any $t \in(0,1)$. The strict convexity of the function $|x|^{p}$ for $p>1$ implies

$$
\begin{aligned}
I(t u+(1-t) v) & =\left(\int_{\mathcal{X}}|\nabla(t u+(1-t) v)-f|^{p} d x\right) \\
& =\left(\int_{\mathcal{X}}|t(\nabla u-f)+(1-t)(\nabla v-f)|^{p} d x\right) \\
& <t\left(\int_{\mathcal{X}}|\nabla u-f|^{p} d x\right)+(1-t)\left(\int_{\mathcal{X}}|\nabla v-f|^{p} d x\right) \\
& =t I(u)+(1-t) I(v)
\end{aligned}
$$

for any $u \neq v$ and $t \in(0,1)$. The equality here is a consequence of the fact that $\nabla u-f$ and $\nabla v-f$ differ from each other on a subset of $\mathcal{X}$ of positive measure. For if they coincide almost everywhere in $\mathcal{X}$, then the difference $u-v$ is constant in $\mathcal{X}$, and so it is actually zero, since $u-v$ vanishes at the nonempty set $\mathcal{S}$.

As already mentioned, this lemma is used in [Mor66] to deduce the lower semicontinuity of the functional $I(u)$. 
Lemma 3.3. The functional $I(u)$ is weakly sequentially lower semicontinuous on $\mathcal{A}$, i.e.,

$$
I(u) \leq \lim _{\nu \rightarrow \infty} \inf I\left(u_{\nu}\right)
$$

for any sequence $\left\{u_{\nu}\right\}_{\nu=1}^{\infty} \subset \mathcal{A}$ converging weakly in $W^{1, p}(\mathcal{X})$ to $u$.

Proof. See Theorem 4.1.1 of [Mor66].

We are now in a position to prove the main result of this section.

Theorem 3.4. The variational problem

$$
I(u) \mapsto \min
$$

over the set $\mathcal{A}$ of all functions $u \in W^{1, p}(\mathcal{X})$ satisfying $u=u_{0}$ at $\mathcal{S}$ is uniquely solvable.

Proof. Since the functional $I(u)$ is bounded from below by 0 , it has infimum $m$ over $\mathcal{A}$. By the definition of infimum, there is a sequence $\left\{u_{\nu}\right\}$ in $\mathcal{A}$, such that $I\left(u_{\nu}\right) \searrow$ $m$. Any such sequence is called minimising. Each subsequence of a minimising sequence is also a minimising sequence. Were it possible to extract a subsequence $\left\{u_{\nu_{\iota}}\right\}$ converging to an element $u \in \mathcal{A}$ in the $W^{1, p}(\mathcal{X})$ norm, then $I\left(u_{\nu_{\iota}}\right)$ would converge to $I(u)=m$, and so $u$ would be a desired solution of our variational problem. It is possible to require the convergence of a minimising sequence in a weaker topology than that of $W^{1, p}(\mathcal{X})$. However, the functional $I$ should be lower semicontinuous with respect to correspondingly more general types of convergence.

In order to find a convergent subsequence of a minimising sequence, one uses a compactness argument. The space $W^{1, p}(\mathcal{X})$ is reflexive. Hence, each bounded sequence in $W^{1, p}(\mathcal{X})$ has a weakly convergent subsequence. Thus, any bounded minimising sequence $\left\{u_{\nu}\right\}$ has a subsequence $\left\{u_{\nu_{\iota}}\right\}$ which converges weakly in $W^{1, p}(\mathcal{X})$ to some function $u$. By a theorem of Mazur, see [Yos65] and elsewhere, any convex closed subset of a reflexive Banach space is actually weakly closed. As we have seen in the proof of Lemma 3.2, the set $\mathcal{A}$ is convex and obviously closed, thus it follows that the limit function $u$ satisfies $u=u_{0}$ on $\mathcal{S}$, i.e., it belongs to $\mathcal{A}$. Moreover, Theorem 3.4.4 of [Mor66] says that the subsequence $\left\{u_{\nu_{\iota}}\right\}$ converges also strongly in $L^{2}(\mathcal{X})$ to $u$. In view of Lemma 3.3

$$
I(u) \leq \lim _{\nu \rightarrow \infty} \inf I\left(u_{\nu}\right)=m
$$

Since $u \in \mathcal{A}$, it follows that

$$
I(u)=m=\inf _{u \in \mathcal{A}} I(u) .
$$

To prove the uniqueness of the solution, consider two different minimizers $u$ and $v$, then $\frac{u+v}{2} \in \mathcal{A}$ and by Lemma 3.2

$$
I\left(\frac{u+v}{2}\right)<\frac{1}{2} I(u)+\frac{1}{2} I(v)=m,
$$

a contradiction.

Corollary 3.5. The mixed boundary value problem (2.1) has a unique solution $u \in W^{1, p}(\mathcal{X})$. 
Proof. The existence of a solution to the mixed boundary problem (2.1) follows from Theorem 3.4. In general, the Euler equations can have solutions which do not provide minima of the functional $I(u)$. However, in our case the correspondence between solutions of the Zaremba problem (2.1) and solutions of the variational problem is one-to-one.

Let $u$ be a solution to the mixed boundary problem (2.1). We would like to show that $u$ is a minimiser for the functional $I(u)$. We use a convexity argument. If $q$ is a convex differentiable function on $\mathbb{R}^{n}$ and $x \in \mathbb{R}^{n}$ fixed, then the inequality

$$
q(\tilde{x}) \geq q(x)+\nabla q(x)(\tilde{x}-x)
$$

holds for all $\tilde{x} \in \mathbb{R}^{n}$. It says that the graph of $q$ lies above each tangential hyperplane. For the convex function $q(x)=|x|^{p}$, where $p>1$, we get

$$
|\nabla w-f|^{p} \geq|\nabla u-f|^{p}+p|\nabla u-f|^{p-2}(\nabla u-f, \nabla w-\nabla u) .
$$

for all $w \in \mathcal{A}$. Integrating over $\mathcal{X}$ we obtain

$$
\int_{\mathcal{X}}|\nabla w-f|^{p} d x \geq \int_{\mathcal{X}}|\nabla u-f|^{p} d x+\int_{\mathcal{X}} p|\nabla u-f|^{p-2}(\nabla u-f, \nabla(w-u)) .
$$

As the difference $w-u$ vanishes on $\mathcal{S}$ and $u$ is a solution of problem (2.1), we obtain

$$
\int_{\mathcal{X}}|\nabla w-f|^{p} d x \geq \int_{\mathcal{X}}|\nabla u-f|^{p} d x
$$

Finally, this shows that $I(w) \geq I(u)$ for all $w \in \mathcal{A}$, i.e., $u$ is a solution of our variational problem.

\section{The Dirichlet to Neumann operator}

In this section we describe the solvability of the Cauchy problem (0.1) using the so-called Dirichlet to Neumann operator, cf. [LT11]. The Dirichlet to Neumann operator is introduced to describe those Cauchy data $u_{0} \in W^{1 / p^{\prime}, p}(\mathcal{S})$, where $1<$ $p<\infty$ and $p^{\prime}=p /(p-1)$, for which the Cauchy problem $(0.1)$ has at least one solution $u \in W^{1, p}(\mathcal{X})$. As mentioned in Section 1, this operator was introduced under additional assumptions (including $d f=0)$ in $([$ AT14]), see Lemma 7.1 there. Now, using Corollary 3.5 we may introduce the Dirichlet to Neumann operator also in the case where $f$ fails to be closed.

Having granted the unique solvability of problem (2.1), we can introduce a Dirichlet to Neumann operator for the Cauchy problem $(0.1)$. Pick $u_{0} \in W^{1 / p^{\prime}, p}(\mathcal{S})$. By Corollary 3.5, there is a unique function $u \in W^{1, p}(\mathcal{X})$ satisfying (2.1). Set

$$
\Psi\left(u_{0}\right):=|\nabla u-f|^{p-2}(\nabla u-f, \nu)
$$

at $\mathcal{S}$, the restriction on $\mathcal{S}$ being understood in an appropriate sense clarified in [Tar95, 1.1.3]. Then, we get $\Psi\left(u_{0}\right) \in W^{-1 / p^{\prime}, p^{\prime}}(\mathcal{S})$.

The following lemma gives a necessary condition for the solvability of the equation $\nabla u=f$ in $\mathcal{X}$.

Lemma 4.1. In order there might exist a function $u \in W^{1, p}(\mathcal{X})$ satisfying $\nabla u=f$ in $\mathcal{X}$ it is necessary that

$$
(f, g)_{L^{2}\left(\mathcal{X}, \mathbb{R}^{n}\right)}=0
$$

for all $g \in W^{1, p^{\prime}}\left(\mathcal{X}, \mathbb{R}^{n}\right)$ satisfying $\operatorname{div} g=0$ in $\mathcal{X}$ and $\nu(g)=0$ at the boundary. 
Proof. The proof of this lemma is based on the Green formula of [Tar90, 2.4.2]. We still identify the gradient operator with exterior derivative $d$ and the adjoint of the gradient operator (which is the divergence operator) with the adjoint $d^{*}$ of $d$ on differential forms. This implies that

$$
\begin{aligned}
(f, g)_{L^{2}\left(\mathcal{X}, \mathbb{R}^{n}\right)} & =(d u, g)_{L^{2}\left(\mathcal{X}, \mathbb{R}^{n}\right)} \\
& =\left(u, d^{*} g\right)_{L^{2}(\mathcal{X})}+(t(u), \nu(g))_{L^{2}(\partial \mathcal{X})} \\
& =0
\end{aligned}
$$

for each $g \in W^{1, p^{\prime}}\left(\mathcal{X}, \mathbb{R}^{n}\right)$ satisfying $d^{*} g=0$ in $\mathcal{X}$ and $\nu(g)=0$ at the boundary.

Having a proper condition for the solvability of $d u=f$ in $\mathcal{X}$, we use the Dirichlet to Neumann operator $\Psi\left(u_{0}\right)$ to describe the set of corresponding Cauchy data at $\mathcal{S}$.

Corollary 4.2. Let $f \in L^{p}(\mathcal{X})$ and $u_{0} \in W^{1 / p^{\prime}, p}(\mathcal{S})$, where $1<p<\infty$. The Cauchy problem (0.1) has a solution $u \in W^{1, p}(\mathcal{X})$ if and only if $f$ satisfies condition (4.1) and $\Psi\left(u_{0}\right)=0$.

Proof. Necessity. If the Cauchy problem has a solution $u \in W^{1, p}(\mathcal{X})$, then $u$ satisfies $\nabla u=f$, and so (4.1) is fulfilled for $f$. Moreover, $u$ is a solution of problem (2.1), hence the definition of the Dirichlet to Neumann operator yields $\Psi\left(u_{0}\right)=0$, as desired.

Sufficiency. Suppose $f \in L^{p}\left(\mathcal{X}, \mathbb{R}^{n}\right)$ satisfies the condition $(f, g)_{L^{2}\left(\mathcal{X}, \mathbb{R}^{n}\right)}=0$ for all $g \in W^{1, p^{\prime}}\left(\mathcal{X}, \mathbb{R}^{n}\right)$, such that $\operatorname{div} g=0$ in $\mathcal{X}$ and $(\nu, g)=0$ at $\partial \mathcal{X}$, and $\Psi\left(u_{0}\right)=0$ at $\mathcal{S}$.

By definition, there exists $u \in W^{1, p}(\mathcal{X})$ such that

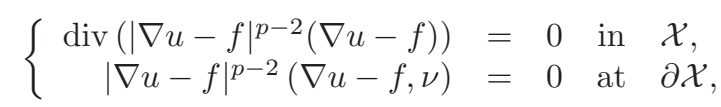

When identifying the gradient operator $\nabla$ with exterior derivative $d$, we think of div as formal adjoint $d^{*}$ of $d$ on differential forms. Consider $g=|d u-f|^{p-2}(d u-f)$, then (4.3) yields

$$
\left\{\begin{array}{c}
d^{*} g=0 \quad \text { in } \quad \mathcal{X} \\
\nu(g)=0
\end{array}\right.
$$

whence

$$
\begin{aligned}
(f, g)_{L^{2}\left(\mathcal{X}, \mathbb{R}^{n}\right)} & =(f-d u, g)_{L^{2}\left(\mathcal{X}, \mathbb{R}^{n}\right)}+(d u, g)_{L^{2}\left(\mathcal{X}, \mathbb{R}^{n}\right)} \\
& =\left(f-d u,|d u-f|^{p-2}(d u-f)\right)_{L^{2}\left(\mathcal{X}, \mathbb{R}^{n}\right)} \\
& =-\|d u-f\|_{L^{p}(\mathcal{X})}^{p} \\
& =0
\end{aligned}
$$

which implies that $d u=f$ and $u=u_{0}$ at $\mathcal{S}$, i.e., the Cauchy problem has a solution.

Note that there is a trick related to a special choice of $g$ which allows one to reduce the proof of Corollary (4.2) to the corresponding result of Section 7 of [AT14]. 


\section{REFERENCES}

[AH96] Adams, D. R., and Hedberg, L., Function Spaces and Potential Theory, Springer, Berlin et al., 1996.

[AT14] Alsaedy, A., and Tarkhanov, N., Normally solvable nonlinear boundary value problems, Nonlinear Analysis 95 (2014), 468-482.

[Cal80] Calderón, A. P., On an inverse boundary value problem, In: Seminar on Numerical Analysis and its Applications to Continuum Physics (Rio de Janeiro, 1980), 1980, 6573 .

[LT11] Ly, I., and Tarkhanov, N., The Dirichlet to Neumann operator for nonlinear elliptic equations, Contemporary Mathematics 553 (2011), 115-126.

[Mor66] Morrey, Charles B., Multiple Integrals in the Calculus of Variations, Springer-Verlag, Berlin et al., 1966

[She13] Shestakov, I., On the Zaremba problem for the $p$-Laplace operator, In: Complex Analysis and Dynamical Systems V, Contemporary Mathematics, vol. 591, Amer. Math. Soc., Providence, RI, 2013.

[Tar90] Tarkhanov, N., Complexes of Differential Operators Kluwer Academic Publishers, Dordrecht, NL, 1995.

[Tar95] Tarkhanov, N., The Cauchy prblem for solutions of elliptic equations, Akademie-Verlag, Berlin, 1995.

[Yos65] Yosida, K., Functional Analysis, Springer-Verlag, Berlin et al., 1965.

[Zar10] Zaremba, S., Sur un problème mixte à l'équation de Laplace, Bull. de l'Académie des sciences de Cracovie, Classe des sciences mathématiques et naturelles, série A, (1910), 313-344.

(Ammar Alsaedy) Universität Potsdam, Institut für Mathematik, Karl-Liebknecht Str. 24/25, 14476 Potsdam, Germany

E-mail address: alsaedy@math.uni-potsdam.de

Department of Mathematics, College of Science, Alnahrain University, Baghdad, IRAQ

E-mail address: ajm@sc.nahrainuniv.edu.iq 